\title{
Analysis of motion for a rolling disc on the flat surface
}

\begin{abstract}
Background: Recent investigations in gyroscopic effects have demonstrated that their origin has more complex nature that represented in known publications. Actually, on a gyroscope are acting simultaneously and interdependently eight inertial torques around two axes. This torques is generated by the centrifugal, common inertial and Coriolis forces as well as the change in the angular momentum of the masses of the spinning rotor. The action of these forces manifests in the form of the inertial resistance and precession torques of gyroscopic devices. New mathematical models for the inertial torques acting on the spinning rotor demonstrate fundamentally different approaches and results of solving the problems of gyroscopic devices. The tendency in contemporary engineering is expressed by the increasing of a velocity of rotating objectslike turbines, rotors, discs and othersthat lead to the proportional increase of the magnitudes of inertial forces forming their motions. This work considers a typical example for computing of the inertial torques acting on the free rolling disc, which can be a bicycle wheel, rims, hoops, discs, and similar designs that express the gyroscopiceffects.
\end{abstract}

Keywords: gyroscope theory, torques, motions, forces
Volume 4 Issue 5 - 2018

Ryspek Usubamatov

Department of Automation\& Robotics, Kyrgyz State Technical University, Kyrgyzstan

Correspondence: Ryspek Usubamatov, Department of Automation\& Robotics, Kyrgyz State Technical University, , 66 Aitmatov Avenue, KSTU, 720044 Bishkek, Kyrgyzstan, Tel +996 3I2 545I25, Email rispek0701@yahoo.com

Received: May 16, 2018 | Published: October 15, 2018

\section{Nomenclature}

$m$ - Mass of a disc

$g$ - Gravity acceleration

$i$ - Index for axis $o x$ or $o y$

$J$ - Mass moment of inertia of a disc

$J_{i}$ - Mass moment of inertia of a disc around axis $i$

$l$-Radius of the disc rolling along the curvilinear path

$R$ - Radius of a disc

\section{$T$ - Load torque}

$T_{a m . i} T_{c t i,} T_{c r i,} T_{i n . i}$ - Torque generated by the change in the angular momentum, centrifugal, Coriolis and common inertial forces respectively, and acting around axis $i$

$T_{r i,} T_{p i}-$ Resistance and precession torque respectively acting around axis $i$

$t$-Time

$\gamma$ - Angle of inclination of a disc

$\eta$ - Coefficient of correction

$\omega$ - Angular velocity of a disc

$\omega_{i}$ - Angular velocity of precession around axis $i$

\section{Introduction}

Most of the textbooks of machine dynamics and publications that dedicated to gyroscope theory content the typical examples with solving of gyroscopic effects by defined analytical approaches. ${ }^{1-3}$ However, the practice demonstrates that the known mathematical models for acting forces on the gyroscopic devices do not match their actual forces and motions. ${ }^{4,5}$ Recent investigations in the area of the physical principles of gyroscopic effects have presented the new mathematical models of forces acting on a gyroscope ${ }^{6-8}$ It is defined that the action of the external load on a gyroscope generates several inertial resistance and precession torques based on the action of the rotating mass elements of the spinning rotor. The resistance torque is generated by the action of the centrifugal and Coriolis forces of the gyroscope's mass elements. The precession torque is generated by the action of the common inertial forces of the gyroscope's mass elements and by the change in the angular momentum of the spinning rotor. These torqueses are acting simultaneously, interdependently and strictly perpendicular to each other around their axes. Equations of inertial torques are represented in Table $1 .^{6}$ Table 1 contains the following symbols and components of the equations that marked by subscript signs indicating the axis of action: $J=m R^{2} / 2$ is the rotor's mass moment of inertia around the spinning axle; $m$ is the mass of the rotor; $R$ is the external radius of the rotor; $\omega$ is the angular velocity of a rotor around axis $i$ and $\omega$ is the angular velocity of a spinning rotor; $T_{r x}$ is the resistance torque acting around axis $o x, T_{p y}$ is the precession torque acting around axis $o y ; T_{c t . i,}, T_{i n . i,} T_{c r . i}$ and $T_{a m . i}$ is the torque acting around axis $i$ that generated by the centrifugal, common inertial forces, Coriolisforces and the angular momentum respectively. This work presents the mathematical model for the free motion of the tilted rolling disc on the flat surface under the action of the eight inertial torques around two axes. Practically, the design of the disc can be the wheels, rims, hoops, discs, and similar designs that possess gyroscopic properties.

\section{Methods}

The simple design of the wheel or thin disc is unstable on the vertical plane, but a rolling motion demonstrates its stability and steering itself in case of the disc tilts. This tilts leads to the turn of the rolling disc to the direction of its fall. This motion of the inclined thin 
disc is the demonstration of the gyroscopic effects, which presented by the action of centrifugal, common inertial, and Coriolis forces and the rate change of the angular momentum. The action of these forces enablesfor bringingback of the rolling disc to a vertical location and allowskeeps its right way rolling. The study of the action of the inertial forces and motion of a rolling thin disc on the flat surface is assumed that the thin disc rolls with the constant angular velocity. Figure 1 represents the rolling disc that slightly tilted on the flat surface. This tilt causes its variable travel on a curved path. The inclination of the rolling disc produces the torque generatedby the gyroscope weight. In turn, the action of this torque produces the following inertial torques (Table 1):

Table I Equations of the gyroscope's inertial torques

\begin{tabular}{|c|c|}
\hline Type of torque generated by & Equation \\
\hline $\begin{array}{l}\text { Centrifugal forces, } T_{c t i} \\
\text { Common inertial forces, } T_{i n . i}\end{array}$ & $T_{c t . i}=T_{i n . i}=2\left(\frac{\pi}{3}\right)^{2} J \omega \omega_{i}$ \\
\hline Coriolis forces, $T_{c r i}$ & $T_{c r}=(8 / 9) J \omega \omega_{i}$ \\
\hline Change in angular momentum, $T_{\text {am. }}$ & $T_{a m . \mathrm{i}}=J \omega \omega_{i}$ \\
\hline Resistance torque $T_{r . i}=T_{c t . i}+T_{c r . i}$ & $T_{r . i}=\left[2\left(\frac{\pi}{3}\right)^{2}+\frac{8}{9}\right] J \omega \omega_{i}$ \\
\hline Precession torque $T_{p . i}=T_{i n, i}+T_{a m . i}$ & $T_{\rho . i}=\left[2\left(\frac{\pi}{3}\right)^{2}+1\right] J \omega \omega_{i}$ \\
\hline $\begin{array}{l}\text { Ratio of the angular velocities } \\
\text { of precesions around two axes }\end{array}$ & $\omega_{2}=\left(4 \pi^{2}+17\right) \omega_{1}$ \\
\hline
\end{tabular}

a. The resistance torques based on the action of the centrifugal $T_{c t . x}$ and Coriolis forces $T_{c r . x}$ acting around axis $o x$.

b. The precession torques based on the action of the change in the angular momentum $T_{a m . x}$ of the rolling disc and the inertial forces $T_{\text {in.x }}$ acting around axis $o y$.

c. The action of the precession torques $T_{a m x}$ and $T_{i n x}$ generates the resistance torques based on the action of the centrifugal $T_{c t . y}$ and Coriolis forces $T_{c r . y}$ acting around axis $o y$.

d. The combined torques acting around axis oy generate the precession torques based on the action of the change in the angular momentum $T_{a m,}$ and the inertial forces $T_{i n,}$ that acting around axis $o x$ and adding to the action of the resistance torques $T_{c t . x}$ and $T_{c r . x}$.

e. The precession torques acting around axis oy turn the rolling disc to the direction of the tilting and the disc rolls by the curve path with the variable radius $l$. The curvilinear motion of the disc generates the action of the centrifugal force of the disc's centre mass that acting horizontally and creating the torque about the contact point of the disc with the surface. This torque acts in the same direction as the combined resistance torques around axis $o x$ that altogether bring the disc to a vertical location.
Figure 1 demonstrates the action of the external and inertial torques on the rolling disc that moves on the flat surface. The mathematical models for the motions of the rolling disc are similar to the models presented. ${ }^{7}$ The action of torques mentioned above around axes $o x$ and oy and represented by the following equations in Euler's form:

$$
\begin{gathered}
T_{c t . m y}-\left(T_{c t . x}+T_{c r . x}+T_{i n . y} \cos \gamma+T_{a m . y} \cos \gamma\right) \eta \\
J_{x} \frac{d \omega_{x}}{d t}=T \cos \gamma-J_{y} \frac{d \omega_{y}}{d t}=T_{i n . x} \cos \gamma+ \\
T_{a m . x} \cos \gamma-T_{c t . y} \cos \gamma-T_{c r . y} \cos \gamma-T_{c r . m y} \\
\omega_{y}=(4 \pi+17) \omega_{x}
\end{gathered}
$$

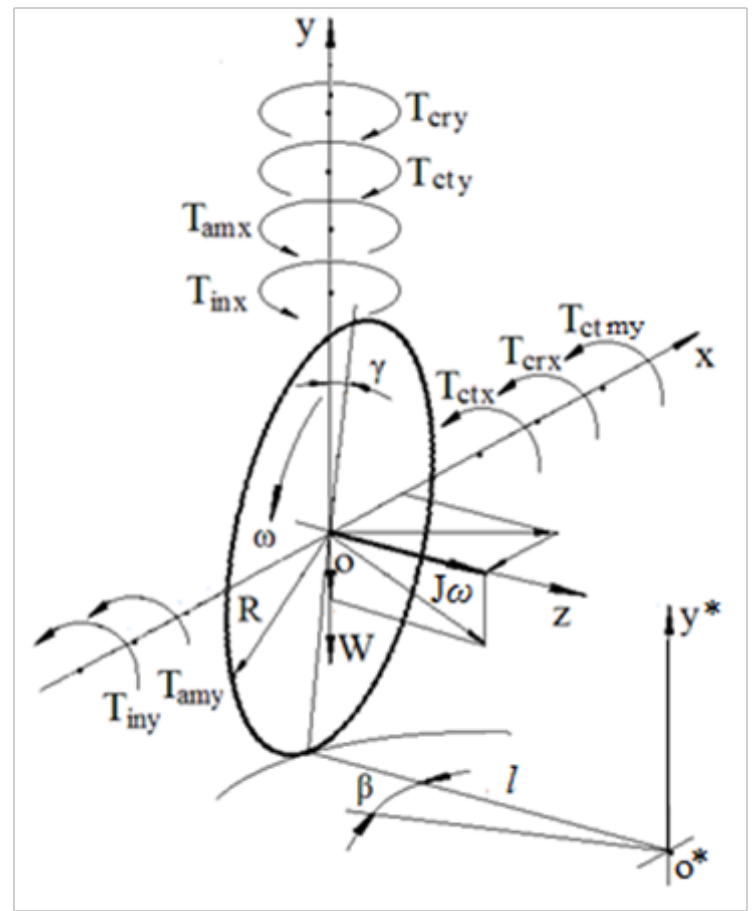

Figure I The torques acting on the free rolling disc on a flat surface.

where $J_{x}=J=m R^{2} / 4+m R^{2}$ is the mass moment of the disc inertia around axis ${ }^{y}$ ox and oy respectively that calculated by the parallel axis theorem; $\omega_{x}$ and $\omega_{y}$ is the angular velocity of precession around axis $o x$ and $o y$ respectively; $T=m g R \sin \gamma$ is the torque generated by the disc weight around axis $o x ; T_{c t, m v}$ is the torque generated by the centrifugal forces of the centre-mass of the rolling disc around axis oy. $T_{\text {ct.x }}$ and $T_{\text {ct.y }}$ is the resistance torque generated by centrifugal forces of the disc mass elements around axes $o x$ and $o y$ respectively; $T_{i n . x}$ and $T_{i n . y}$ is the precession torque generated by inertial forces around axes $o x$ and $o y$ respectively; $T_{c r y}$ and $T_{c r y}$ is the resistance torque generated by Coriolis forces around axes $o x$ and $o y$ respectively; $T_{a m . x}$ and $T_{a m . y}$ is the precession torque generated by the change in the angular momentum of the spinning rotor around axes $o x$ and $o y$ respectively; $T_{c r m y}$ is the torque generated by Coriolis force of the rotating gyroscope centremass around axes $o x$ and $o y ; \eta$ is the correction coefficient. The torque generated by the centrifugal force of the centre-mass of the rolling disc around axis $o y^{*}$ defined by the following equation: 


$$
T_{c t, m y}=F_{c t . m y} R \cos \gamma=m \omega \omega_{x}(4 \pi+17)(R \cos \gamma)^{2}
$$

Where $F=m l \cos \gamma \cdot(V / l)^{2}=m \cos \gamma(\omega R)^{2} / l$ is the centrifugal force of the disc centre-mass rotating around axis $o y^{*}, m$ is the disc mass. The variable radius $l$ of the disc rolling around axis $o y^{*}$ is defined from the expressions $\omega R=\omega_{y} l$ and $\omega_{y}=(4 \pi+17) \omega_{x}$ , then, $l=\omega R /\left[(4 \pi+17) \omega_{x}\right]$; other components ${ }^{y}$ are as specified above. The torque generated by Coriolis force of the centre-mass of the rolling disc around axis $o y^{*}$ and the motion along the axis $o z$ in the negative direction defined by the following equation:

$T_{c r, m y}=F_{c r . m y} \cdot l \cos \gamma=m l \omega_{x} \sin \gamma \cdot(\omega R / l) \cdot l \cos \gamma=m(\omega R)^{2} \cos \gamma \sin \gamma$

where $F=m l \omega \sin \gamma \cdot(\omega R / l)$ is Coriolis force of the disc centre-mass rotating around axes $o x$ and $o y^{*}, V=l \omega \sin \gamma$ is the tangential velocity of the disc centre-mass around $^{x}$ axis $o x ; \omega=\omega R / l$ is the angular velocity of the disc centre-mass around axis $\stackrel{m}{o y^{*}}$; and $\omega_{x}$ and $\omega_{y}$ is the angular velocity of the precession of the disc around axes ox and oy respectively; other components are as specified above. The action of the Coriolis torque around axes oy decreases the magnitude of the combinedinertial torquesacting around axis $o y$. Hence, the magnitude of the inertial torques acting around axis $o x$ also is decreased proportionally due to their interdependency. The changes in values of inertial torques acting around two axes are equal because the inertial torques are expressed by the inertial kinetic energies of the rolling disc that are equal along axes. Then, the mathematical model for the correction coefficient $\eta$ is represented by the following equation:

$\eta=\frac{\left(T_{\text {in. } x}+T_{a m . x}\right) \cos \gamma-T_{\text {cr. } m y}}{\left(T_{\text {in. } x}+T_{\text {am. }}\right) \cos \gamma}=1-\frac{T_{c r . m y}}{\left(T_{\text {in. } . x}+T_{a m . x}\right) \cos \gamma}=1-\frac{m \omega R^{2} \sin \gamma}{\left[2\left(\frac{\pi}{3}\right)^{2}+1\right] J \omega_{x}}$

Substituting defined parameters (Eqs. (4) - (6)) and equations of the torques (Table 1) into Eqs. (1) and (2) and transformation yields the following system of differential equations:

$$
\begin{aligned}
& J_{x} \frac{d \omega_{x}}{d t}=m g R \sin \gamma-m \omega \omega_{x}(4 \pi+17)(R \cos \gamma)^{2}- \\
& {\left[2\left(\frac{\pi}{3}\right)^{2} J \omega \omega_{x}-\frac{8}{9} J \omega \omega_{x}-J \omega \omega_{y} \cos \gamma\right] \times\left\{1-\frac{m \omega R^{2} \sin \gamma}{\left[2\left(\frac{\pi}{3}\right)^{2}+1\right] J \omega_{x}}\right\}}
\end{aligned}
$$

Analysis of Eq. (7) demonstrates the similarity to the equations of the gyroscope motions suspended from the flexible cord. ${ }^{7}$ Hence, the solution of Eq. (7) is the same. Then, the equation for the angular velocity of the disc around axis $o x$ is represented by the following expression:

$$
\begin{aligned}
& J_{x} \frac{d \omega_{x}}{d t}=m g R \sin \gamma-m \omega \omega_{x}\left(4 \pi^{2}+17\right)(R \cos \gamma)^{2}- \\
& {\left[2\left(\frac{\pi}{3}\right)^{2}+\frac{8}{9}+4 \pi^{2}+17\right] J \omega \omega_{x} \cdot\left\{1-\frac{m \omega R^{2} \sin \gamma}{\left[2\left(\frac{\pi}{3}\right)^{2}+1\right] J \omega_{x}}\right\}}
\end{aligned}
$$

where all parameters are as specified above.

Solving Eq. (8) enables for computing the angular velocity of the precession around axis oxand around axis oy (Table 1) for the rolling disc on the flat surface. Additionally, the numerical solution of the right side of Eq. (8) will show that inertial torques of the rolling disc always turn up the inclined rolling disc to the vertical location.

\section{Working example}

The mathematical model for the motion of the rolling discon the flat surface is considered with the following example whose data is presented in Table 2. The acting external and internal torques on the disc presented in Figure 1. The spinning disc initially possesses an inclined axle.It is necessary to find the magnitudes of the internal torques exerted on the rolling disc and its precessions.

Table 2 Technical data of the thin disc

Parameter Data

Angular velocity, $\omega$

$2.0 \mathrm{rad} / \mathrm{s}$

Radius of the disc, $R$

Angle of tilt, $\gamma$

Weight, $m$

Mass moment

Around axis oz, $\mathrm{J}=\mathrm{mR}^{2} / 2$

of

Around axes ox and oy of the centre mass,

inertia, $\mathrm{kgm}^{2}$

$\mathrm{J}=\mathrm{mR}^{2} / 4$

Around axes ox and oy at the point of support, $\mathrm{Jx}=\mathrm{Jy}=\mathrm{mR}^{2} / 4+\mathrm{mR}^{2}$

Equation (8) is transformed and simplified that yields the following expression:

$$
\begin{aligned}
& J_{x} \frac{d \omega_{x}}{d t}=m \sin \gamma\left[g R+\left(\frac{38 \pi^{2}+161}{2 \pi^{2}+9}\right)(\omega R)^{2}\right]- \\
& {\left[m\left(4 \pi^{2}+17\right)(R \cos \gamma)^{2}+\left(\frac{38 \pi^{2}+161}{9}\right) J\right] \omega \omega_{x}}
\end{aligned}
$$


Substituting given data into Eq. (9) yields the following:

$0.015 \frac{d \omega_{x}}{d t}=0.5 \times \sin 10^{\circ} \times\left[9.81 \times 0.2+\left(\frac{38 \pi^{2}+161}{2 \pi^{2}+9}\right) \times(2.0 \times 0.2)^{2}\right]-$ $\left[0.5 \times\left(4 \pi^{2}+17\right) \times\left(0.2 \times \cos 10^{\circ}\right)^{2}+\left(\frac{38 \pi^{2}+161}{9}\right) \times 0.01\right] \times 2.0 \omega_{x}$

Separating variables and presenting in the integral form gives the following:

$$
\int_{0}^{\omega_{x}} \frac{d \omega_{x}}{0.126975628895-\omega_{x}}=225.481762368666 \int_{0}^{t} d t
$$

Solving the integral equations yields the following equation:

$$
\ln \left(0.126975628895-\omega_{x}\right) \mid \begin{aligned}
& \omega_{x}=-225.481762 t \\
& 0
\end{aligned}
$$

Giving rise to the following result:

$$
\ln \left(\frac{0.126975628895-\omega_{x}}{0.126975628895}\right)=-225.481762 t \text { or }
$$

$$
\omega_{x}=0.126975628895\left(1-e^{-225.481762 t}\right)
$$

Where the expression $e^{-225.481762 t} \approx 0$ and can be neglected.

Then the angular velocities of precession for the rolling disc are as follows:

$$
\begin{aligned}
& \omega_{x}=0.126975628 \mathrm{rad} / \mathrm{s} \\
& \omega_{y}=\left(4 \pi^{2}+17\right) \omega_{x}=7.171382594 \mathrm{rad} / \mathrm{s}
\end{aligned}
$$

The angular velocity $\omega_{y}$ of the rolling disc around axis oy leads to the turn of the disc to the side of its tilting and decreasing of this angle. The presented data of a tilted rolling disc allow considering the magnitudes of the acting torques. Computing the right side of Eq. (8) demonstrates that the value of the inertial torques (second component) is always bigger than the first one and presented by the followingresult:

\section{$0.429460328682<0.429460328696$}

This result is the validation that the inertial torques of the rolling disc turns up the disc until the vertical location.

\section{Results and discussion}

New analytical approach to the inertial forces acting on the gyroscopic devices enables for developing the equations of the torques and motionsof any rotating objects moving in the space. The mathematical models derived for the motionof the rolling disc on the flat surface based on the action of the centrifugal, common inertial and Coriolis forces, as well as the change in the angular momentum. The new analytical approach to the gyroscope problems demonstrates and explains the physical principles of acting forces on a rolling disc and its motions. The mathematical model for the motion of the rolling disc on the flat surface confirms the gyroscope properties by the practical observation and represents a good example of the educational process.

\section{Conclusion}

In the area of publications of gyroscopic effects, the motion of the rolling disc is one of the most complex and intricate in terms of analytical solutions. Known mathematical models for the rolling disc motions are accepted with simplifications and do not adequately express a real picture of its behaviour. The new mathematical models for gyroscope torques consider the simultaneous and interdependent action of several inertial forces generated by the rotating mass elements and centre mass of the spinning rotor. As a practical application, these new physical principles for gyroscope motions were used for modelling of the free rolling motion of the inclined disc. This mathematical model is distinguished from the well-known publications, which are solving by the numerical modelling that does not interpret the physical origin of gyroscopic effects. The application of new mathematical models for the free rolling of the inclined disc on the flat surface clearly demonstrates physical principles of acting forces.

\section{Acknowledgements}

None.

\section{Conflict of interest}

The author declares there is no conflict of interest.

\section{References}

1. Hibbeler RC, Yap KB. Mechanics for engineers - statics and dynamics. $13^{\text {th }}$ ed. Prentice Hall Pearson, editor. Singapore; 2013.

2. Gregory DR. Classical mechanics. Cambridge University Press; New York, USA. 2006.

3. Aardema MD. Analytical dynamics. Theory and Application. Academic/ Plenum Publishers; New York, Springer. 2005.

4. Liang WC, Lee SC. Vorticity, gyroscopic precession, and spin-curvature force. Physical Review. 2013;D 87:044024.

5. Zhang N, Ren YF, Li SK. Research on testing method of dynamic characteristicfor MEMS-gyroscope. Advanced Materials Research. 2012;346:515-520.

6. UsubamatovR. Inertial forces acting on gyroscope. Journal of Mechanical Science and Technology. 2018;32(1):101-108.

7. Usubamatov R. A mathematical model for motions of gyroscope suspended from flexible cord. Cogent Engineering. 2016;3(1).

8. Usubamatov R. Analysis of motions for gyroscope with one side support. Sch J Appl Sci Res. 2018;1:5-9. 